\title{
An Integrated Adoption Model to Manage Blockchain-Driven Business Innovation in a Sustainable Way
}

\author{
Nusi Drljevic ${ }^{1, *}$, Daniel Arias Aranda ${ }^{1}\left[\right.$ and Vladimir Stantchev ${ }^{2}$
}

1 Department of Business and Economics, University of Granada, 18071 Granada, Spain; darias@go.ugr.es

2 Institute of Information Systems, SRH University Berlin, 10587 Berlin, Germany; vladimir.stantchev@srh.de

* Correspondence: nusi@correo.ugr.es

check for

updates

Citation: Drljevic, N.; Aranda, D.A.; Stantchev, V. An Integrated Adoption Model to Manage Blockchain-Driven Business Innovation in a Sustainable Way. Sustainability 2022, 14, 2873. https://doi.org/10.3390/su14052873 Academic Editors: Rainer Lasch and Jacob Lohmer

Received: 31 December 2021 Accepted: 27 February 2022 Published: 1 March 2022

Publisher's Note: MDPI stays neutral with regard to jurisdictional claims in published maps and institutional affiliations.

Copyright: (C) 2022 by the authors. Licensee MDPI, Basel, Switzerland. This article is an open access article distributed under the terms and conditions of the Creative Commons Attribution (CC BY) license (https:// creativecommons.org/licenses/by/ $4.0 /)$.

\begin{abstract}
Blockchain technology has the potential to drive innovations across various industries, businesses, and use cases. It is broadly recognized that innovation is a vital source of competitive advantage in a rapidly changing environment. High expectations surround blockchain's potential for contributing to sustainable economic and social development. However, current blockchain projects still show high failure rates. The associated side effects of such failure rates generate a negative impact on economic and social sustainability performances such as corporate governance, risk management, finance management, human resources and culture management, and competitiveness. This paper assesses adoption models and their risk and success factors. Building on a novel, integrated adoption model to operationalize, measure and manage blockchain-driven business innovation in a sustainable way, we assessed its applicability with an empirical study across 20 industry sectors and 125 business leaders. The results reveal that the developed adoption model holds the potential to support the sustainable usage of blockchain technology for business innovations, not limited to a specific industry or use case. Further case studies and industry activities can be carried out to continue its validation in future works.
\end{abstract}

Keywords: blockchain; sustainable blockchain; blockchain adoption; technology adoption; blockchain innovation; business innovation; sustainable operations; requirements engineering

\section{Introduction}

Blockchain technology holds the promise to empower the design of innovative business models, operating models, products, services, and consumer experiences. It is broadly recognized that innovation is a vital source of competitive advantage in a rapidly changing environment. Innovation is both a process and an outcome [1]. Like with foundational technologies of the past, business innovation through blockchain requires change at the system level in order to accelerate the wider adoption of blockchain technology. Despite broad interest in blockchain applications, there are still high failure rates and low maturity levels across industry sectors. Missing standardization and requirements engineering approaches are reasons for the failure of most blockchain projects [2].

Until 2019, 92\% of blockchain projects failed due to a lack of existing models for managing the adoption of blockchain-driven business innovation in a sustainable way [3]. By mid-2021, still, 2047 blockchain and cryptocurrency projects have failed [4]. This high failure rate speaks for the broader complexity of technology-powered change [5]. The associated side effects of such failure rates often have a negative impact on economic and social sustainability performances such as corporate governance, risk management, finance management, human resources and culture management, and competitiveness. Although there are currently $70 \mathrm{M}$ active blockchain wallets, mostly used to trade cryptocurrency transactions within the decentralized finance and gaming industry, it appears that broad industry-wide blockchain adoption is still in its infancy $[6,7]$. Due to the gaps of the first generation blockchains, today, it is estimated that $90 \%$ of blockchain enterprise 
platforms need to be replaced with newer blockchains to increase scalability and speed. The primary reasons why more industries have decided to adopt blockchain technology are business model innovations, value chain innovations, and greater overall security of data management [8].

These challenges and reasons represent a growing demand for managing blockchaindriven business innovation in a sustainable way. Two aspects are affecting this demand, according to Iansiti and Lakhani [9]. The first aspect is a novelty, i.e., the degree to which an approach is not seen before. Novel approaches require more effort to explain what problems they solve. The second aspect is complexity, represented by the level of ecosystem coordination involved: the number and diversity of parties that need to work together to produce value with the technology. In applying these two aspects to blockchain, the authors argue in their Harvard Business Review report that adoption of this technological innovation will take years. They expect that the sum of total blockchain applications will not reach broad adoption and critical mass for at least another decade and probably more [9].

We advance that managing the aspects of novelty and complexity in an integrated way is a key for sustainable industry-wide blockchain adoption. As a solution, we propose an integrated adoption model, a combination of the "Technology Acceptance Model" (TAM) and the "Capability Maturity Model" (CMM), that can support a sustainable acceleration of blockchain technology adoption.

Adoption is understood as a process of innovating, and the relevant aspects of this process are acceptance and maturity. Acceptance is a decision-based process, which is determined by user attitudes, values, and users' intention to use a system or a technology [10]. Maturity can be understood as various stages of evolution, which users move through in complex environments. It shows the degree to which any novel aspects of a business are applied across the process [11]. Together, the integration of acceptance and maturity (TAM and $(\mathrm{CMM}$ ) describe and manage user behaviors and stages throughout the innovation process and are therefore key for sustainable and successful blockchain adoption.

There is a general consensus in the industry and the society that both a focused discussion and a standardization framework for requirements management in the relatively young blockchain business context has yet to emerge [12], and we seek to highlight, advance, and contribute to the discourse in this domain. This assumption could be rooted in hypotheses such as: (1) stakeholders in the business innovation space are eager to apply a model that foster the sustainable adoption of blockchain technology in the context of business innovation; (2) stakeholders in the business innovation space depend upon risk and success factors that can be standardized and operationalized in order to sufficiently manage the blockchain-driven innovation process. An example of such a standard is the ISO/IEC/IEEE 29148 standard [13]. This standard applies a life cycle-based approach to define processes and products in the context of requirements engineering.

This paper identifies a selection of existent theories and models for managing technology adoption and presents a new adoption model (TAM + CMM) to manage requirements engineering with risk and success factors for the sustainable usage of blockchain-driven business innovation. The remainder of the article is structured as follows: Section 2 presents our materials and methods. There we also formulate four research questions. Section 3 maps our results to the four research questions. The discussion of our results and their applicability toward blockchain, risk management, and sustainability are presented in Section 4. Finally, Section 5 provides a conclusion and an outlook on future research activities.

\section{Materials and Methods}

Following from the hypotheses, sources were sought out, which specifically address adoption and its two resilient expressions: acceptance, which is tightly linked to user attitudes and behavioral intention, and maturity, e.g., the various stages of evolution, which users in business contexts move through during the adoption process [10]. Acceptance 
and maturity level management are critical to adopting, implementing, and using any new technology. Numerous models and theories exist for understanding and tracking both acceptance and maturity, and we seek to identify and introduce those with the potential to standardize and operationalize risk and success factors for blockchain-driven business innovation.

\subsection{This Paper Considers Four Main Research Questions (RQ)}

RQ1. How is adoption defined within business and technology contexts, and why is it relevant?

RQ2. Which theories and adoption models are available for requirements engineering of blockchain-driven business innovation?

RQ3. What are the current research gaps in the area of blockchain adoption and the requirements engineering for sustainable blockchain-driven business innovation?

RQ4. Which new adoption model can empower blockchain-driven business innovation in a sustainable way by operationalizing acceptance and maturity level criteria?

\subsection{Methods and Approach}

In the context of this work, we applied a quantitative secondary research method first. Existing works were assessed using the method of a systematic literature review (SLR). Publications were sourced from the databases and citation indexes Web of Science, Elsevier's Science Direct, SSSR, Research Gate, and Academia.edu using methods such as keywording across titles and abstracts. The fast pace of the topic in industry led us to focus on sources from the last five years and to also consider relevant industry sources (soc. gray literature).

The keywords that we applied to the search were: "acceptance models", "adoption models", "blockchain applications", "blockchain adoption”, "blockchain innovation", "maturity models", and "sustainable blockchain". Our expectation was to pinpoint relevant approaches for managing blockchain-driven business innovation, which in turn will provide answers to the research questions. Regardless of the fast-paced activities around blockchain in industry, the number of academic publications specifically dealing with blockchain adoption models is not high.

Furthermore, we have conducted an empirical study across 125 business leaders and 20 industry sectors. For this purpose, we employed questionnaires and expert interviews. The selection of study participants was designed to ensure broad representation of companies acting in various industry sectors. At the personal level, we aimed to include experts from various levels of responsibility within the relevant organizations.

\section{Results}

Following the method proposed in Section 2, we conducted our literature review primarily in the summer and fall of 2021 and did a validation at the end of the same year. The initial search conducted on Elsevier's Science Direct database yielded 2559 results for "blockchain applications and adoption", with an increase in publication volume from five articles in 2016 to 1185 articles in 2021. In total, 387 publications are reviewed publications. A subsequent review of the 387 results showed that 36 publications have the keywords "blockchain and adoption" in the title and were directly relevant for answering our RQs and relied on the frequency of viewings, citations, and sharing as a means of assessing their importance [14].

The further search conducted on Elsevier's Science Direct database yielded 3413 results for "sustainable blockchain", with an increase in volume from 9 articles in 2016 to 1585 articles in 2021. In total, 440 publications are reviewed publications. A subsequent review of the 440 results showed that 40 publications have the keywords "sustainable blockchain" in the title and were directly relevant for answering our RQs and relied on the frequency of viewings, citations, and sharing as a means of assessing their importance [15] 
However, most of these academic publications focus on industry-specific use cases such as healthcare, supply chain, aviation, banking, logistics, and IoT [16-21]. An integrated adoption model including risk and success factors to foster a sustainable adoption of blockchain technology across various industries was unavailable.

Our systematic literature review and empirical study were successful in providing answers to the four research questions (RQ) that we formulated in Section 2. Below, we present the results of each research question.

\subsection{RQ1: How Is Adoption Defined within Business and Technology Contexts and Why Is It Relevant?}

Adoption is understood as a process of innovating, and the key aspects of this process are "acceptance" and "maturity". The degree of adoption is characterized by the extent to which a given technology becomes accepted and incorporated into approved business practices [22].

Acceptance is defined as a favorable decision for the use of any technology, product, or process based upon behavioral factors [23]. It is determined by user attitudes and values, and it is tightly coupled to behavioral intention [10].

Maturity is defined as the stages of evolution toward the full operationalization of technological processes [24]. Maturity is the degree to which any novel aspect of a business or organization is adopted and applied [25].

While acceptance is a decision-making process based upon users' behavior and beliefs, maturity is a dynamic process of evolution, which is related to ongoing business operations.

Hence adoption is the act of taking on something new as one's own. In business innovation, adoption is the process by which any novel application, operation, or product is accepted, implemented, optimized, and further used. The aspect of novelty is inherent to innovation, and basically, anything that is new to a company qualifies as innovation [26]. In the context of business and technology, the term adoption is linked to innovation, which can be a complex process due to the number of parties being involved.

Innovation is widely regarded as a critical source of competitive advantage in an increasingly changing environment [1], synthesizing the following meaning from literature published on innovation over the past 27 years: "Innovation is production or adoption, assimilation, and exploitation of a value-added novelty in economic and social spheres; renewal and enlargement of products, services, and markets; development of new methods of production; and the establishment of new management systems. It is both a process and an outcome" [27].

To activate and realize any innovation, it must be adopted and consistently managed over time. In their discussions of the metrics of innovation, both Edison et al. [26] stress the importance for companies to continually innovate, or else they tend to lose their position to emerging companies with innovative offerings.

As a result, business leaders, decision makers, designers, developers, investors, and other stakeholders in the blockchain-driven business innovation space must understand why users accept a particular system or not, as well as how users reach higher levels of maturity with that system by applying risk and success factors that enable a standardized approach to operationalize and manage requirements engineering sustainably. The goal is to achieve stages of evolution toward the full operationalization of technology-driven innovation processes.

In this context, it is mission-critical to apply risk and success factors to operationalize blockchain-driven business innovation in a sustainable way [2]. These factors include agility, architecture, costs, governance, interoperability, scalability, vision, and others. These decision-making risk and success factors are part of the user experience, and they must be taken into account throughout the technology's development phases and entire life cycle [28]. Only then favorable decisions for the use of new business models, operating models, products, services, or processes based upon behavioral factors can be matured 
in a way where the given technology becomes accepted and incorporated into new and approved business practices [26].

\subsection{RQ2: Which Theories and Adoption Models Are Available for Requirements Engineering of Blockchain-Driven Business Innovation?}

Adoption models and frameworks are required to navigate the novelty and complexity of foundational technologies such as blockchain. As described above, novelty and complexity are strongly related to acceptance and maturity. These aspects influence the successful and sustainable adoption of blockchain-driven business innovations. Understanding what motivates stakeholders to decide for or against technological innovation makes it possible to gauge how and why any particular technology will gain market traction or not. Understanding how a technology-driven innovation reaches maturity is central to the company's management process.

This systematic literature review identifies a selection of existent theories and adoption models for managing technology adoption. Table 1 shows a choice of well-established theories as well as integrated models for managing technology adoption. The review of the following models shall provide valuable insights for creating a new adoption model for sustainable blockchain-driven business innovation: "Theory of Reasoned Action" (TRA), "Diffusion of Innovation Theory" (DOI), "Motivational Model" (MM), "Theory of Use and Gratification" (U\&G), "Technology Acceptance Model" (TAM), "Capability Maturity Model" (CMM), as well as two younger examples of integrated models such as "Technology Acceptance Model" and "Diffusion of Innovation Theory" (TAM + DOI), and "Technology Acceptance Model" and "Theory of Planned Behavior" (TAM + TPB).

Table 1. Theories and adoption models.

\begin{tabular}{ll}
\hline Theories and Models & Functions and Parameters \\
\hline $\begin{array}{l}\text { Theory of Reasoned } \\
\text { Action (TRA) }\end{array}$ & $\begin{array}{l}\text { Prediction of human behavior via three cognitive } \\
\text { components: (1) attitudes, (2) subjective norms, (3) } \\
\text { behavioral intentions [29]. }\end{array}$ \\
\hline & $\begin{array}{l}\text { Four factors to measure the rate of any innovation's } \\
\text { adoption: (1) the innovation itself, (2) communication } \\
\text { channels, (3) time, (4) social system. Five innovation } \\
\text { parameters: (1) compatibility, (2) relative advantage, } \\
\text { (3) complexity, (4) trialability, (5) observability [30]. }\end{array}$ \\
\hline $\begin{array}{l}\text { Motivational Models } \\
\text { (MM) }\end{array}$ & $\begin{array}{l}\text { Two forms of motivation to adopt and use technology: } \\
\text { (1) extrinsic motivation, (2) intrinsic motivation [31]. }\end{array}$ \\
\hline Technology Acceptance Model (TAM) & $\begin{array}{l}\text { Five two determinants for technology acceptance in } \\
\text { the field of information technology (IT): (1) perceived } \\
\text { usefulness, (2) perceived ease of use, attitude toward } \\
\text { using, behavioral intention to use (BI), actual use (USE) } \\
\text { [10]. }\end{array}$ \\
\hline $\begin{array}{l}\text { Process-based model designed across five phases: (1) } \\
\text { initial, ad hoc processes, (2) repeatable, (3) defined, (4) } \\
\text { managed, (5) optimizing [24]. }\end{array}$ \\
\hline Model (CMM) \\
$\begin{array}{l}\text { Integrated Model } \\
\text { (TAM + DOI) }\end{array}$ \\
$\begin{array}{l}\text { See determinants from above “DOI" and "TAM" } \\
\text { [32,33]. }\end{array}$ \\
\hline $\begin{array}{l}\text { Look up five determinants perceived ease of use, } \\
\text { perceived usefulness, attitude, subjective norm, and } \\
\text { perceived behavioral control [34]. }\end{array}$ \\
\hline
\end{tabular}

The "Theory of Reasoned Action" (TRA) was developed by Martin Fishbein and Icek Ajzen in 1967. This theory of attitudes and behavior was a key source for the later developed "Technology Acceptance Model" (TAM). "TRA" is used to predict and explain any human behavior through three main cognitive components: attitudes (favorable or unfavorable 
feelings), subjective norms (social influence according to several factors such as society, economy, politics, demographic factors, etc.), and behavioral intentions (motivation to decide for or against any action). The theory suggests that a person's behavioral intentions (BI) will depend on his attitudes and subjective norms [35].

Rogers' "Diffusion of Innovation Theory" (DOI) is a model that seeks to explain how, why and at what rate new ideas and technology spread [30]. Innovations in terms of ideas, practices, or objects are perceived as new by those adopting and using them. Furthermore, diffusion is discussed as the process by which an innovation is communicated through certain channels over time among the members of a social system. Therefore, the "DOI" theory argues that potential users make decisions to adopt or reject an innovation based on beliefs that they form about the innovation [36]. The model introduces four factors to measure the rate of any innovation's adoption: the innovation itself, communication channels, time, and social system. The rate of adoption is expressed in terms of the percentage of innovation. This value is then categorized across five lifecycle stages, according to five adopter types: innovators, early adopters, early majority, late majority, and laggards. "DOI" also highlights the point at which any technology reaches the state of critical mass [30] and tries to explain the innovation decision process by predicting the likelihood and rate of adoption of an innovation [37]. In essence, "DOI" is a dynamic model that measures the evolution of innovation among different users over time.

Numerous "Motivational Models" (MM) are available, and these derive from motivation theory and the field of motivation research, which has developed since the 1940's. The theory works with two forms of motivation to adopt and use a technological innovation: extrinsic motivation and intrinsic motivation. Extrinsic motivation describes the desire to achieve some outcome distinct from the technology itself, e.g., using the technology for the purpose of improved job performance. Intrinsic motivation drives the use of the technology simply for the purpose of the activity, per se [31].

The "Technology Acceptance Model" (TAM) is an information system theory that was initially published with the aim to improve the understanding of technology acceptance and gain insights into the design and implementation of information systems. "TAM" is the most widely applied model of users' acceptance and usage of technology. It evolves "TRA's" multitude of parameters with two key parameters for technology acceptance: perceived usefulness (PU) and perceived ease of use (PEU). External variables determine the values of these two parameters, which then influence the attitude of the user, which can be assessed as either favorable or unfavorable with regard to technological innovations. Perceived usefulness directly influences behavioral intention (BI) to use a technological system. This, in turn, determines the actual use of the system [38].

Developed by Carnegie Mellon University's Software Engineering Institute (SEI), the "Capability Maturity Model" (CMM) describes the development of capabilities. It was originally developed to meet the needs and characteristics of governmental organizations. In 1986, the SEI began to develop a maturity framework that would help developers


which is used to measure a company's current maturity level with any innovation. This enables users to identify strengths and improvement points and accordingly prioritize what to do in order to reach higher maturity levels [39]. People, organizations, operations, processes, etc., evolve through a process of growth and development in the direction of more advanced maturity, going through distinct levels. The model's structure is most commonly based on five maturity levels: (1) initial, (2) repeatable, (3) defined, (4) measured, (5) optimized [24]. Levels in the model serve as bases on which higher maturity levels can be planned and implemented. The aim of maturity models is to quantify the activities carried out, make them measurable and develop them (i.e., allow them to mature) over time [40].

The design of a "CMM" can range from very general to highly specified. Over the past three decades, numerous standardized models have been developed, and these ready-made models are generally adopted by businesses, whereas tailored models have been created 
for specific applications. A systematic mapping study of 237 articles shows that current research into maturity models at the time was applicable to more than 20 domains, heavily dominated by software development and software engineering [11].

In the context of blockchain adoption, there are various maturity model approaches. Leading global consultancy firm KPMG devised a blockchain maturity model based upon eight areas of identified risk, which could influence the rate of blockchain adoption. The risk areas include (1) access and user management, (2) authorization and provisioning management, (3) data management, (4) interoperability, (5) scalability and performance, (6) change management, (7) privacy, and (8) security [28].

Wang et al. provide a maturity model for blockchain technology, identifying these 14 areas of risk to be managed across 5 stages of maturity: (1) network load, (2) reliability, (3) architecture, (4) upgrading, (5) integration, (6) maintenance, (7) storage, (8) scalability, (9) business efficiency, (10) standardization, (11) computational complexity, (12) privacy, (13) data security, and (14) transaction security. Additionally, the authors apply four indicators of technology maturity, as defined by the Association for Computing Machinery's ACM Computing Classification System: (1) networks, (2) information systems, (3) computing methodologies, and (4) security and privacy. Each risk area is assigned a lower or higher level of maturity, displaying their assessment of the state of blockchain maturity at the time of the study [25]. The authors conclude that their model serves as a guide for more systematic decision making regarding blockchain adoption. An assessment of the current state of the product (e.g., blockchain application) by an organization is necessary prior to adoption into a strategic plan. Despite the emerging importance of blockchain maturity and accessibility, the authors recognize that little research into assessing blockchain maturity has been covered in previous literature. They argue for the importance of measuring maturity because if you cannot measure it, you cannot manage or improve it [25].

Two examples from the literature employ the methodology of merging the "TAM" and "DOI" models. In both cases, the models hold that the five innovative characteristics (compatibility, complexity, relative advantage, trialability, and observability) have an effect on the users' perceived use (PU), perceived ease of use (PEU), and behavioral intention to use (BI) technological innovations. These characteristics are a key aspect of the diffusion of innovation. The degree to which innovation is regarded as being compatible with the potential end-users' values, prior experiences, and needs is referred to as compatibility. Complexity is the end-users' perceived level of difficulty in understanding innovations and their ease of use. Relative advantage is defined as the degree to which an innovation is considered as being better than the idea it replaced. This construct is found to be one of the best predictors of the adoption of an innovation. Trialability refers to the degree to which innovations can be tested on a limited basis. Observability is the degree to which the results of innovations can be visible by other people [32].

Lou and Li advanced the integrated "TAM" and "DOI" model for the initial purpose of supporting employees' intentions to use e-learning systems. The model suggests an integrated "TAM" and "DOI" model in assessing the adoption of blockchain technology from the business manager's perspective. Their research aims to deliver and test a unified framework for investigating the continual process of blockchain technology adoption [32].

A further model that aims to intertwine "TAM" with the "Theory of Planned Behaviour" (TPB) was proposed by Safeena et al. for the purpose of Internet banking adoption [19]. Here, authors "marry" the "TPB" model from the social psychology field with "TAM". Perceived usefulness and perceived ease of use are two significant factors affecting how an information system or a new technology is accepted. The authors found a positive relationship between perceived ease of use and perceived usefulness to be critical factors for the use of e-banking. 
3.3. RQ3: What Are the Current Research Gaps in the Area of Blockchain Adoption and the Requirements Engineering for Sustainable Blockchain-Driven Business Innovation?

Due to the relatively young research field of blockchain technology adoption and, most importantly, the sustainable usage of blockchain technology for business innovation and business operation, there appears a range of research gaps. In this context, we would like to highlight four major gaps:

Our initial search showed the meager output of publications related to keywords "sustainable blockchain usage" and "blockchain adoption models". Moreover, research and documentation of the impact of blockchain projects are lacking, thus creating a gap in content to support awareness, knowledge, implementation, scale, and optimization as maturity practices of sustainable blockchain usage. This demonstrates a clear need for standards and requirements management in this area.

Furthermore, there is currently no normative, universally applicable adoption model available, which can empower organizations and stakeholders to accelerate and manage blockchain-driven business innovation in a sustainable way. We derive this gap from identifying that each of the sources either focuses on specific use cases such as healthcare, supply chain, aviation, banking, logistics, and IoT with certain criteria or the existing theories and models capture only parts of the requirements engineering and innovation process [16-21]. This gap reflects a clear need to design an integrated adoption model that can empower stakeholders across any industry and use case to use blockchain technology for sustainable business innovation.

The third identified gap is a normative and consensus-generated framework of risk and success factors that can be embedded in an adoption model in order to operationalize, measure, manage and optimize requirements engineering for business innovation and operations in a sustainable way.

The fourth gap within the scope of this research is a lack of applied projects and studies that are made available for the community and all stakeholders that are eager to innovate business models and business operations with the help of blockchain technology. Due to the distributed and decentralized nature of blockchain technology, it would be mandatory to provide such a learning environment in order to accelerate blockchain-driven innovation across any industry. More matured industries such as "Gaming" or "Finance" could provide valuable insights to other, less matured industries.

While we could locate some studies on integrated adoption models, combining "TAM" with "DOI" or "TAM" with "TPB", further research and development initiatives could support the active testing of these integrated models, for example, with industry-wide blockchain projects. There is a lack of merging existing adoption models to manage the aspects of novelty and complexity in the context of blockchain technologies. An integrated model for managing both aspects would be beneficial for a better understanding of acceptance and maturity as key aspects of the blockchain adoption process in the context of business innovation.

\subsection{RQ4: Which New Adoption Model Can Empower Blockchain-Driven Business Innovation in a} Sustainable Way?

\subsubsection{Design of the Novel, Integrated "TAM + CMM Adoption Model"}

As discussed above, the two aspects that influence blockchain adoption are novelty and complexity [11]. We advance that managing the aspects of novelty and complexity in an integrated way is a key for sustainable and industry-wide blockchain adoption. In order to work with these aspects simultaneously and close the identified research gaps, we propose an integrated adoption model, a combination of the "Technology Acceptance Model" (TAM) and the "Capability Maturity Model" (CMM) to manage and accelerate blockchain-driven business innovation sustainably.

Novelty is linked to acceptance, and complexity is linked to maturity. Acceptance is a decision-based process, which is determined by user attitudes, values, and users' intention to use blockchain technology $[10,41]$. Maturity is understood as various stages of 
evolution of new technology adoption through different stages, which users move through in complex environments.

Based on the conceptual framework as seen in Figure 1, we designed the novel "TAM + CMM Adoption Model", which includes seven levels on the x-axis and six levels on the y-axis.

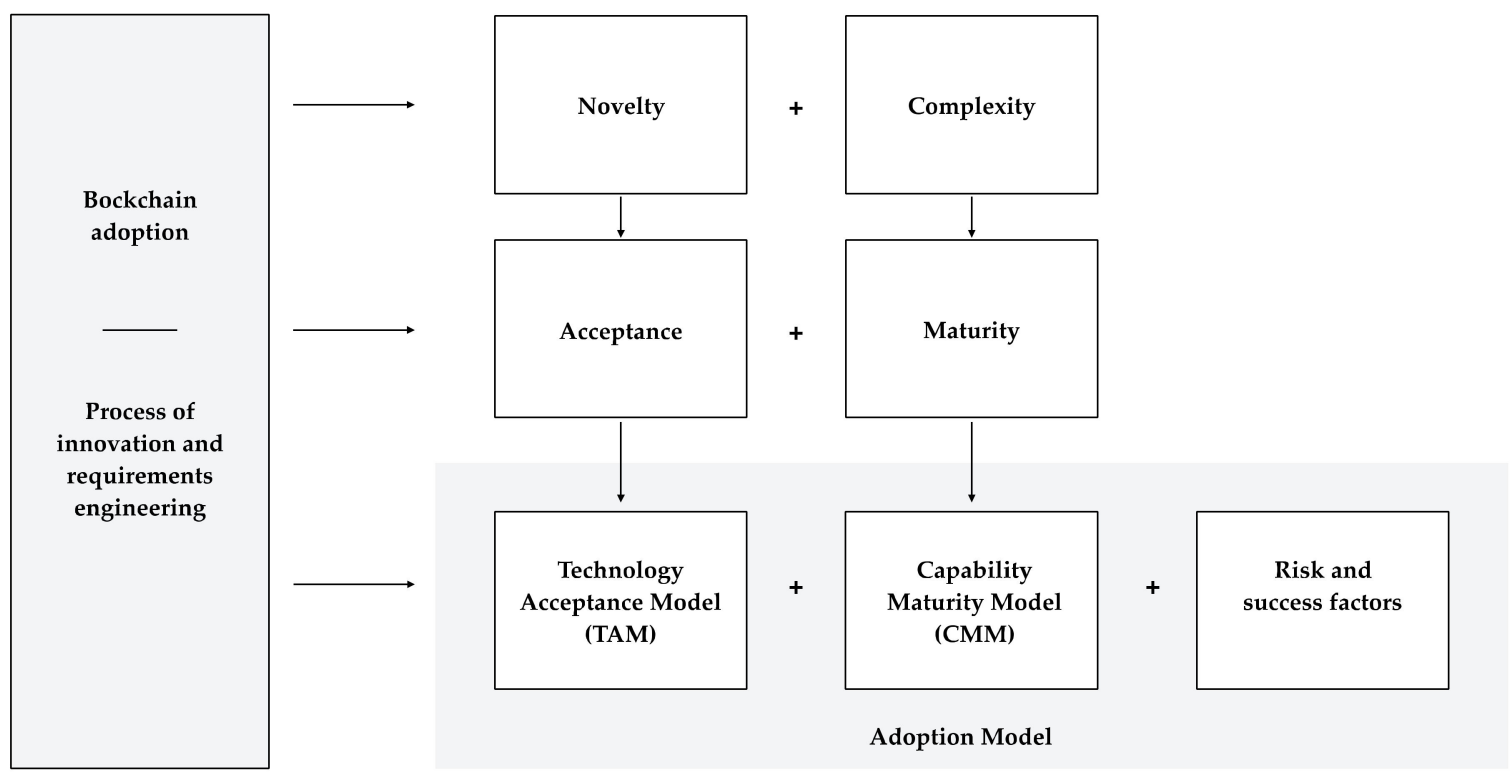

Figure 1. Conceptual framework for the new, integrated blockchain adoption model.

The "TAM" model was extended to include the parameters "level of knowledge" (LK) and "perceived risk" (PR). In line with the aspect of novelty, we added these parameters in order to provide a more complete picture of acceptance, taking early stages of awareness and knowledge into consideration along with the subjective uncertainty surrounding the technology in question.

"Level of knowledge" (LK) was added as a parameter for assessing acceptance because this is an important signifier of how much users already know about the technology and, in turn, how much facilitating activity is needed to create awareness of the technology's value. "Perceived risk" (PR) is a risk value based upon uncertainty of the outcomes of deciding for or against blockchain technology. Stakeholders and users have several goals or expectations when accepting a novel technology, and the possibility of these goals and expectations not being met is represented by a degree of perceived risk.

The "CMM" model was extended to include the parameters "awareness" (level 1) and "knowledge" (level 2). Awareness of blockchain technology is mandatory and a prerequisite for adoption. The degree of knowledge about a technology influences the adoption rate. In line with the dimension of complexity, we added these parameters in order to provide a more complete picture of understanding blockchain technology, its values, and benefits before initiating a project. Creating awareness for blockchain technology and its risk and success partners and educating stakeholders within this space is mission-critical to reduce complexity and foster a sustainable maturity process for the requirements engineering.

As shown in Figure 2, the "TAM" parameters shown on the y-axis have been partly extended from the original model to include a total of six parameters. From top to bottom, the following definitions apply to blockchain technology acceptance. "Level of knowledge" (LK) refers to how aware users are of blockchain technology. Some degree of knowledge about a technological system can be understood as a prerequisite to acceptance. "Perceived usefulness" (PU) is a parameter of motivation to accept and use blockchain technology. If users do not perceive blockchain technology as useful, they are unlikely to accept and use it. "Perceived risk" (PR) is the level of subjective uncertainty among users when deciding 
whether to accept blockchain technology. The possibility that blockchain technology will not live up to users' expectations is represented by "perceived risk". "Perceived ease of use" (PEU) is a crucial factor that influences sustainable acceptance and usage of blockchain technology. Any system must be perceived as easy-to-use, or it is less likely to be accepted by users. Whereas "perceived usefulness" (PU) deals with blockchain technology's usefulness or relevance, "perceived ease of use" (PEU) is a measure of how operable or easy a technology is perceived to be. "Behavioral intention to use" (BI) refers to the degree of concrete activities to use blockchain technology. This indicates a clear intention and a relatively advanced stage of acceptance. "Actual system use" (ASU) refers to the technology or aspect of the technology that is currently in use. This is an indicator of the actual level of acceptance. If a system is active in use, it has already been accepted.

\section{Capability maturity model (CMM)}

\begin{tabular}{|c|c|c|c|c|c|c|c|}
\hline & $\begin{array}{l}\text { Awareness } \\
=\text { level } 1\end{array}$ & $\begin{array}{c}\text { Knowledge } \\
=\text { level } 2\end{array}$ & $\begin{array}{l}\text { Initiation } \\
=\text { level } 3\end{array}$ & $\begin{array}{c}\text { Implementation } \\
=\text { level } 4\end{array}$ & $\begin{array}{l}\text { Standardization } \\
\quad=\text { level } 5\end{array}$ & $\begin{aligned} & \text { Scale } \\
= & \text { level } 6\end{aligned}$ & $\begin{array}{l}\text { Optimization } \\
\quad=\text { level } 7\end{array}$ \\
\hline $\begin{array}{l}\text { Level of } \\
\text { knowledge } \\
\text { = LK }\end{array}$ & & & & & & & \\
\hline $\begin{array}{l}\text { Perceived } \\
\text { usefulness } \\
\quad=\mathrm{PU}\end{array}$ & & & & & & & \\
\hline $\begin{array}{l}\text { Perceived risk } \\
\quad=\mathrm{PR}\end{array}$ & & & & & & & \\
\hline $\begin{array}{l}\text { Perceived ease } \\
\text { of use } \\
=\text { PEU }\end{array}$ & & & & & & & \\
\hline $\begin{array}{l}\text { Intention to us } \\
\quad=B I\end{array}$ & & & & & & & \\
\hline $\begin{array}{l}\text { Active } \\
\text { system use } \\
=\text { ASU }\end{array}$ & & & & & & & \\
\hline
\end{tabular}

Figure 2. Customized, integrated adoption model "TAM" and "CMM" to empower sustainable blockchain-driven business innovation. Y-axis = "Technology Acceptance Model” (TAM), X-axis “Capability Maturity Model" (CMM).

With the "Capability Maturity Model" (CMM), we have customized and extended the model to define the seven levels of maturity shown along the x-axis: awareness (level 1), knowledge (level 2), initiation (level 3), implementation (level 4), standardization (level 5), scale (level 6), and optimization (level 7).

Awareness is an indicator of having heard about blockchain technology. The level of awareness increases when blockchain technology becomes more widely diffused. Knowledge of blockchain technology goes beyond awareness and indicates that stakeholders and users have invested in understanding and learning about blockchain technology and its risk and success factors. Awareness about blockchain technology and its risk and success factors alone is not sufficient. Stakeholders need to gain detailed knowledge about blockchain technology and its risk and success factors in order to be empowered to initiate a project and to be equipped to manage the requirements engineering of blockchain-driven innovation in a sustainable way.

Initiation refers to projects that initiate the innovation process by introducing blockchain technology and its risk and success factors into existent business operations.

Implementation is the process of making a project active and effective. At this level of maturity, real innovation begins in terms of changing business models, operations, working processes, habits, and behaviors of all parties involved by using the risk and success factors as criteria for maturity development. 
Once a project has been implemented, these changes and new operations can be tested, and new standards can be developed and defined. The maturity levels take significant progress and become manifested as a used practice.

With establishing standards across the innovation process, the next level of scaling the innovation is by reaching larger user groups within the operations. At this stage, the adoption of blockchain technology is fully matured and accepted.

The highest level of maturity within this model is optimization. At this point, processes of improvement can be carried out to increase functionality or effectiveness.

As described and discussed above, we advance to apply risk and success factors across all levels (x-axis, y-axis) of the "TAM + CMM Adoption Model" to operationalize, measure, and manage blockchain adoption for business innovation in a sustainable way. Neglecting or ignoring risk and success factors means creating risks of failures, which has a negative impact on any type of resources and missing out on market competitiveness. Risk and success factors are mission-critical to deal with the dimensions of novelty and complexity in order to increase acceptance rates and maturity levels within the adoption process [2].

It appears that some risk and success factors in managing blockchain adoption are regularly applied, whereas others are based on specific applications of blockchain technology. In either case, no normative taxonomy of blockchain success factors could be identified. Among the sources reviewed, risk and success factors such as scalability, performance, governance, security, and change management appear to be prevalent. Several works consider the areas of governance or regulatory frameworks as areas of possible weakness or risk. In this context, consultancy firm KPMG names eight areas where risks should be considered: access and user management, authorizing and provisioning management, data management, interoperability, scalability, and performance, change management, privacy, and security [28,42].

The OECD identifies data quality, data security, upfront costs and lack of access, privacy rules and data security, scalability, technical performance, and energy consumption as relevant factors [10]. A further discussion of success factors and risks to blockchain's becoming mainstream defines the lack of user experience (UX) or customer experience (CX) as risk considerations as well as architecture design and system integration [43,44].

In a recent publication, similar risk and success factors and challenges that are relevant for blockchain adoption are discussed, such as data privacy, security, regulatory uncertainty, governance and leadership readiness, transparency, and speed [45].

Based on the above described scientific and practical consensus, retrieved from various publications and studies, Table 2 provides an overview of key risk and success factors. This list is not exhaustive but should provide an overview of risk and success factors to operationalize, measure, and manage blockchain adoption in a sustainable way. Additional factors can be added to the list if a clear value for operationalization and sustainable adoption is visible.

From the above-presented risk and success factors, we choose agility, customer experience, and interoperability to exemplary demonstrate how they are operationalized, measured, and managed within the "TAM + CMM Adoption Model".

Agility is one of the most relevant competitive advantages of companies and organizations in response to the constant changes of transformative and volatile markets. Information technology, including blockchain, requires agility in the development, but it also generates organizational and business agility when active and implemented [23]. Key performance indicators to manage agility are cycle-time, escaped defect-rate, planned-todone ratio, or team health. 
Table 2. Excerpt of risk and success factors within the "TAM + CMM Adoption Model" model, in alphabetical order.

\begin{tabular}{lll}
\hline 01 Agility & 11 Customer experience & 21 Privacy \\
\hline 02 Architecture & 12 Data management & 22 Processes \\
\hline 03 Authorization & 13 Effectiveness & 23 Regulatory \\
\hline 04 Availability & 14 Efficiency & 24 Scalability \\
\hline 05 Capability & 15 Governance & 25 Security \\
\hline 06 Communication & 16 Integration & 26 Speed \\
\hline 07 Compatibility & 17 Interoperability & 27 Strategy \\
\hline 08 Compliance & 18 Legal liability & 28 User experience \\
\hline 09 Costs & 19 Liquidity & 29 Velocity \\
\hline 10 Culture & 20 Performance & 30 Vision \\
\hline
\end{tabular}

Recent research shows specific patterns in executives' use of customer experience key performance indicators. The key is not so much the number of metrics they track but how they use them to grow their businesses by focusing on customer segmentation rate, customer acquisition costs, customer lifetime value, and churn rate [46].

Interoperability denotes that two or more systems or components are able to exchange information without special effort on either system. Interoperability is more than compatibility and less than full integration. Compatibility is a minimum requirement for two systems to interact. A system that is interoperable can be operated independently, but one that is integrated loses its functionality when the flow of services is interrupted. A suite of systems must, of necessity, be interoperable, but interoperable systems need not be integrated [47]. Related key performance indicators are the number of processed files, number of defaults by file, cost of file processing, profitability per contract.

Interoperability and agility are also applied across other domains, not limited to technology only. Interoperability, as well as agility, are equally relevant in domains such as organization, operating model, people and collaboration, and others. It is highly recommended that KPIs are aligned across various domains. Key performance indicators (KPIs) are quantifiable measures a company uses to determine how well it meets its strategic, tactical, and operational goals. The above-defined key performance indicators (KPIs) were identified by using the balanced scorecard (BSC) and further literature within the specific domain [46].

\subsubsection{Testing the "TAM + CMM Adoption Model" with an Empirical Study}

The above-presented "TAM + CMM Adoption Model" was tested with an empirical study. The empirical study was conducted pre-pandemic in 2019 across 20 industries and 125 business leaders and decision makers. The industries include in alphabetical order advertising, automotive, consulting, e-commerce, education, fashion, finance, FMCG (fast-moving consumer goods), healthcare, HR services, information technology, law, manufacturing, logistics, marketing, media, pharma, retail, software, travel, and transportation. Participants of the empirical study span executive positions in business, operation, and technology functions. The companies that participated in this empirical study represent large enterprises, medium businesses, and start-ups. Table 3 shows the share of respondents by leadership role and Table 4 presents the share of respondents by company sizes. 
Table 3. Share of respondents by leadership role.

\begin{tabular}{lll}
\hline Position & $\begin{array}{l}\text { Number of Survey } \\
\text { Respondents }\end{array}$ & $\begin{array}{l}\text { Share of Survey } \\
\text { Respondents }\end{array}$ \\
\hline C-level & 47 & $37.6 \%$ \\
\hline Managing Director & 13 & $10.4 \%$ \\
\hline Senior + Vice President & 8 & $6.4 \%$ \\
\hline Director & 25 & $20 \%$ \\
\hline Head of Department & 14 & $11.2 \%$ \\
\hline Senior Manager & 18 & $14.4 \%$ \\
\hline
\end{tabular}

Table 4. Share of respondents by company sizes.

\begin{tabular}{lll}
\hline Company Size & $\begin{array}{l}\text { Number of Survey } \\
\text { Respondents }\end{array}$ & $\begin{array}{l}\text { Share of Survey } \\
\text { Respondents }\end{array}$ \\
\hline Large businesses (1000+ employees) & 46 & $36.8 \%$ \\
\hline $\begin{array}{l}\text { Medium businesses (100-999 } \\
\text { employees) }\end{array}$ & 31 & $24.8 \%$ \\
\hline Small businesses (1-99 employees) & 48 & $38.4 \%$ \\
\hline
\end{tabular}

We present the relevant part of the empirical study that relates to RQ4. Due to the large scope of the empirical study, we aim to deliver the complete study, including a post-pandemic analysis within a subsequent paper.

The survey consists of 29 predefined, closed-ended questions and 6 open-ended questions: a total of 35 questions. With this uniquely designed survey framework, the sets of parameters from the respective "TAM" and "CMM" models were merged into an integrated model. Each of the 13 parameters was mapped into the survey questions, illustrating which levels of acceptance and maturity are simultaneously analyzed with each question.

To follow the overarching goal of this survey, questions were designed in a way that responses could indicate the levels of acceptance and maturity parameters. In this way, it was possible to identify how ready and equipped business leaders are to apply blockchain technology for business innovation.

The key research questions pursued was: Are business leaders empowered enough with regards to acceptance levels "awareness", "perceived usefulness" (PU), "perceived ease of use" (PEU), "intention to use" (BI), and "active use" (ASU) to use blockchain technology across the seven maturity levels? Are business leaders equipped with the right risk and success factors to manage blockchain-driven business innovation?

Descriptive Analysis

Blockchain technology in the context of business innovation is a relevant area for business leaders. This can be qualified by a high awareness rate, as $91.2 \%$ of respondents have heard about blockchain technology (LK), and $76.8 \%$ of respondents claim to know what blockchain technology is and how it functions (LK).

However, a strong decline in "TAM" parameters (level of knowledge, perceived usefulness, perceived ease of use, intention to use, active system use) across the "CMM" maturity levels, regardless of industry sector or company size, indicates that there is a substantial gap in managing the blockchain adoption process in a sustainable way. Only $5 \%$ of respondents are using a comprehensive methodology to apply blockchain for business innovation. Furthermore, only one out of 125 respondents answered positively across all 7 maturity levels (CMM) and "TAM" parameters. 
Empirical Analysis

We analyzed the status quo of blockchain adoption across a wide range of industries. In particular, business leaders and decision makers from 20 industry sectors responded to questions to blockchain acceptance levels and maturity levels.

The empirical analysis of certain companies within an industry is conducted via the hypotheses $\mathrm{H} 1$ and $\mathrm{H} 2$ and a Fisher test for proportions. The empirical analysis regarding risk measures at the industry scale, as explored with hypothesis $\mathrm{H} 3$, is conducted via a binomial test.

The following hypotheses were tested: (H1) Business leaders are knowledgeable of blockchain technology and its value in terms of perceived usefulness (PU), (H2) business leaders are knowledgeable of blockchain technology, and its value in terms of perceived ease of use (PEU), (H3) the risk measure across analyzed industries, which arises by not using an integrated adoption model with respect to "TAM" and "CMM" when applying blockchain technology is higher than $50 \%$.

We operationalized and measured the perceived usefulness (PU), perceived ease of use (PEU), and the perceived risks (PR) of the defined industry sectors by not adopting blockchain in a sustainable way, as this could have a negative impact in terms of sustainability performances and missing out on innovation and competitive advantage. Due to low positive respondents across "TAM" maturity levels 3-7, the statistical test to measure "TAM" acceptance parameters perceived usefulness (PU), perceived ease of use (PEU), and perceived risk (PR) was conducted on the "CMM" maturity level 2 (level of knowledge).

To test the hypotheses H1 and H2, we applied Fisher's exact test due to the small sample sizes. With Fisher's exact test, one can test whether proportions from two variables are independent of one another.

Equation (1): Fisher test equation

$$
\mathrm{p}=\frac{(\mathrm{a}+\mathrm{b}) !(\mathrm{c}+\mathrm{d}) !(\mathrm{a}+\mathrm{c}) !(\mathrm{b}+\mathrm{d}) !}{\mathrm{a} ! \mathrm{b} ! \mathrm{c} ! \mathrm{d} ! \mathrm{n} !}
$$

The exclamation mark (!) indicates the factorial operator. To test whether the proportions of two variables are independent of one another, which represents the null hypothesis here, one needs to calculate the $p$-value, which is the probability of obtaining the empirical result under the null hypothesis. This $p$-value must be calculated over all extreme cases with the same marginal totals. If this $p$-value is smaller than the common level of significance, one can reject the null hypothesis. The common levels of significance are $0.01,0.05$ and 0.10 , or $1 \%, 5 \%$, and $10 \%$, respectively. Furthermore, we used the two-tailed Fisher's exact test due to interest in testing the mutual independency. In this case, when testing whether answers from two different questions are independent, one can reject the null hypothesis on a common level of significance and conclude that those answers from two questions are statistically independent. The data gathering provides the following answers' distribution across the integrated approach.

H1. The association between the positive answers of question 11 (Q11) and question 13 (Q13) of the questionnaire, as presented in Appendix A, is considered to be statistically significant. This statement is based on $\mathrm{p}$-value $=8.16 \times 10^{-9}$. Hence, we can assume with sufficient statistical security that the answers from both questions are dependent. Thus, business leaders who know about blockchain technology may know about its value in terms of perceived usefulness.

H2. The association between the positive answers of question 11 (Q11) and question 14 (Q14) of the questionnaire, as presented in Appendix A, is considered to be statistically non-significant. This statement is based on $\mathrm{p}$-value $=0.199$. Hence, we can assume with sufficient statistical security that the answers from both questions are independent. Therefore, the statement of business leaders' knowledge of how blockchain functions may not be supported by an appropriate methodology for applying blockchain technology in their company. 
Analyzing the distribution of answers, we could recognize that only a few companies have initiated or implemented a blockchain project. Moreover, there is only one company that has applied and advanced its blockchain project across all maturity levels. Due to this fact, a risk analysis is necessary. The aim is to analyze the measure of risk for each industry sector in terms of gaining or losing competitive advantage as well as managing the associated side effects of blockchain project failure rates that create a negative impact on economic and social sustainability performances such as corporate governance, risk management, finance management, human resources and culture management by not applying a comprehensive adoption model.

Hence, a risk analysis is performed at the industry scale in order to analyze the aggregated levels of PU and PEU within particular industry sectors and the degrees of blockchain technology acceptance among business leaders. Risk and success factors, as presented in Table 2, play a relevant role at each maturity level, and thus, the risk analysis can be carried out at each maturity level. However, the number of responses in the survey allows only for the risk analysis at the CMM level of "knowledge" (level 2), which corresponds to survey questions Q13 for PU and Q14 for PEU as presented in Appendix A.

To test hypothesis H3, we applied a binomial test. The binomial test was applied to test if risk measures are higher than $50 \%$. We can use this test to establish whether the proportion of 'Yes' and 'No' answers within a group is higher than $50 \%$, which yields our hypothesis 3 . The statistic of the binomial test is distributed as follows:

Equation (2): Binomial test equation

$$
P(X=i)=B\left(i \mid p_{0}, n\right)=\left(\begin{array}{c}
n \\
i
\end{array}\right) p_{0}^{i}\left(1-p_{0}\right)^{n-i}
$$

Here $n$ is the number of answers, and $p_{0}$ is the assumed proportion of 'Yes' answers, which are coded with 1 . In our case, we test whether $p_{0}$ is higher than $50 \%: 0.5<p_{0}$. The binomial test is an exact test so that it can be used for small data sets, as in this case. We would like to point out that the risk measure we introduce in Equation (2) is based upon 'No' answers. Hence, testing whether this risk measure is statistically significantly higher than $50 \%$ is equivalent to testing whether the number of ' $\mathrm{No}^{\prime}$ answers is higher than $50 \%$. With the calculation, we only consider industry sectors with two or more responses.

Table 5 presents the data produced by applying the binomial test. The results are organized by the following columns, from left to right: number of the industry sectors (in alphabetical order), name of the industry sectors represented in the survey, number of answers submitted for each industry sector, and the number of positive answers submitted for each industry sector, the risk measure $\mathrm{R}$ and the $p$-values for both perceived usefulness (PU) and perceived ease of use (PEU), respectively.

The $p$-value indicates the level of significance at which the null hypothesis can be rejected. If the $p$-value is lower than $0.10,0.05$, or 0.01 , the null hypothesis can accordingly be rejected at a significance level of $10 \%, 5 \%$, or $1 \%$. Our assumption is that a risk measure of higher than 0.50 or $50 \%$ means that the risk of missing out on blockchain-driven business innovation by not sustainably adopting the technology is higher than the measure of opportunity to realize blockchain adoption. In this case, blockchain project failure rates rise, and the associated side effects that create a negative impact on economic and social sustainability performances such as corporate governance, risk management, finance management, human resources and culture management, and competitiveness increase.

With the $v$-values for PU, the null hypothesis can be rejected for the industry sectors marketing and manufacturing. For all other industry sectors, the null hypothesis cannot be rejected. With PEU's $p$-values, the null hypothesis can be rejected for the industry sectors advertising, consulting, e-commerce, education, fashion, IT, law, manufacturing, marketing, media, pharma, retail, software, and travel. 
Table 5. Analysis of integrated "TAM + CMM Adoption Model" parameters.

\begin{tabular}{|c|c|c|c|c|c|c|c|c|}
\hline & \multirow[b]{2}{*}{$\begin{array}{l}\text { Industry } \\
\text { Sector }\end{array}$} & \multirow[b]{2}{*}{$\begin{array}{l}\text { Number of } \\
\text { Respon- } \\
\text { dents }\end{array}$} & \multicolumn{3}{|c|}{$\begin{array}{l}\text { TAM: Perceived Usefulness (PU) } \\
\text { TAM: Perceived Risk (PR) } \\
\text { CMM: Level of Knowledge (LK) }\end{array}$} & \multicolumn{3}{|c|}{$\begin{array}{l}\text { TAM: Perceived Ease of Use (PEU) } \\
\text { TAM: Perceived Risk (PR) } \\
\text { CMM: Level of Knowledge (LK) }\end{array}$} \\
\hline & & & $\begin{array}{l}\text { Number of } \\
\text { Positive Re- } \\
\text { spondents }\end{array}$ & $\begin{array}{l}\mathbf{R}=\text { Risk } \\
\text { Measure }\end{array}$ & $p$-Value & $\begin{array}{l}\text { Number of } \\
\text { Positive Re- } \\
\text { spondents }\end{array}$ & $\mathbf{R}$ & $p$-Value \\
\hline 1 & Advertising & 8 & 4 & 0.50 & 0.6367 & 1 & 0.88 & 0.0352 \\
\hline 2 & Automotive & 5 & 3 & 0.40 & 0.8125 & 2 & 0.60 & 0.5000 \\
\hline 3 & Consulting & 7 & 4 & 0.43 & 0.7734 & 0 & 1.00 & 0.0078 \\
\hline 4 & E-commerce & 6 & 2 & 0.67 & 0.3437 & 0 & 1.00 & 0.0156 \\
\hline 5 & Education & 7 & 2 & 0.71 & 0.2266 & 0 & 1.00 & 0.0078 \\
\hline 6 & Finance & 2 & 1 & 0.50 & 0.7500 & 0 & 1.00 & 0.2500 \\
\hline 7 & Fashion & 4 & 1 & 0.75 & 0.3125 & 0 & 1.00 & 0.0625 \\
\hline 8 & Healthcare & 3 & 3 & 0.00 & 1.000 & 0 & 1.00 & 0.1250 \\
\hline 9 & $\begin{array}{l}\text { Human } \\
\text { resource }\end{array}$ & 5 & 1 & 0.80 & 0.1875 & 1 & 0.80 & 0.1875 \\
\hline 10 & IT & 11 & 5 & 0.55 & 0.7256 & 2 & 0.82 & 0.0327 \\
\hline 11 & Law & 4 & 2 & 0.50 & 0.6875 & 0 & 1.00 & 0.0625 \\
\hline 12 & Logistics & 4 & 2 & 0.50 & 0.6875 & 1 & 0.75 & 0.3125 \\
\hline 13 & Manufacturing & 9 & 1 & 0.89 & 0.0195 & 0 & 1.00 & 0.0019 \\
\hline 14 & Marketing & 15 & 4 & 0.73 & 0.0592 & 0 & 1.00 & 0.0000 \\
\hline 15 & Media & 9 & 5 & 0.44 & 0.7461 & 0 & 1.00 & 0.0019 \\
\hline 16 & Pharma & 4 & 3 & 0.25 & 0.9375 & 0 & 1.00 & 0.0625 \\
\hline 17 & Retail & 5 & 3 & 0.40 & 0.8125 & 0 & 1.00 & 0.0312 \\
\hline 18 & Software & 7 & 5 & 0.29 & 0.9375 & 0 & 1.00 & 0.0078 \\
\hline 19 & Travel & 6 & 1 & 0.83 & 0.1094 & 0 & 1.00 & 0.0156 \\
\hline 20 & Others & 4 & 2 & 0.60 & 0.5000 & 0 & 1.00 & 0.3125 \\
\hline
\end{tabular}

Looking at the R or risk measures above 50\% within CMM level "knowledge" and TAM level "perceived usefulness" (PU), it appears that the following industry sectors face the risk of missing out on blockchain-driven business innovation as well as risking increased failure rates of blockchain projects: Advertising, e-commerce, education, finance, fashion, human resource, IT, law, logistics, manufacturing, marketing, travel, and others.

Looking at the R or risk measures above 50\% within CMM level "knowledge" and TAM level "perceived ease of use" (PEU), it appears that the following industry sectors face the risk of missing out on blockchain-driven business innovation as well as risking to increase failure rates of blockchain projects: advertising, automotive, consulting, ecommerce, education, finance, fashion, healthcare, human resource, IT, law, logistics, manufacturing, marketing, media, pharma, retail, software, travel, and others.

In summary, the results imply that most of the analyzed companies face risks to manage blockchain-driven business innovation already in early CMM levels such as "knowledge". Due to low positive respondents across "TAM" maturity levels 3-7, the statistical test to measure "TAM" acceptance parameters perceived usefulness (PU), perceived ease of use (PEU), and perceived risk (PR) was conducted on the "CMM" maturity level 2 (level of knowledge) could not be conducted, which implies that the risks are even higher.

\section{Discussion}

Our results provided answers to the formulated research questions. However, these answers and insights should be considered in their appropriate context. With RQ1, our analysis illustrates that adoption can be understood as a process of innovating, and the key phases of this process are the two aspects of novelty and complexity, which are linked 
to acceptance and maturity. While both aspects are sound and relevant for blockchain adoption, there might also be other aspects that could be used to create a conceptual framework for a blockchain adoption model. It shall be highlighted that integration of relevant aspects such as acceptance and maturity is required to navigate the novelty and complexity of this foundational technology for business innovation.

By answering RQ2, we identified various theories and models that could be used to integrate relevant aspects for designing a new blockchain adoption model that enables stakeholders to use blockchain for business innovation in a more sustainable way. The need to integrate aspects such as acceptance and maturity is evident since there is still such a high failure rate in blockchain adoption.

The analysis and identified gaps in the context of RQ3 shows that the overall research in blockchain adoption models and, most importantly, clear operationalization of such adoption models is still young and requires more research. This need becomes evident because there is currently no normative, universally applicable adoption model available, which can empower companies and organizations to accelerate and sustainably manage blockchain-driven business innovation. We derive this gap from identifying that each of the reviewed sources either focuses on specific use cases with certain criteria or the existing theories and models capture only parts of the requirements engineering for the innovation process. This reflects a clear need to design an integrated adoption model that can empower stakeholders across any industry to use blockchain technology for sustainable business innovation.

Answering RQ4, based on the answers of the previous RQ1, RQ2, RQ3 created the opportunity to use the analyzed theories "TAM" and "CMM" as a potential solution to design an integrated blockchain adoption for sustainable business innovation. We advance that managing the aspects of novelty and complexity in an integrated way is a key for sustainable industry-wide blockchain adoption since both user attitudes, values, and intentions as well as various stages of evolution, which users move through in complex innovation environments. Furthermore, it is noticeable that risk and success factors need to be embedded in such an adoption model in order to operationalize, measure, manage and optimize requirements engineering for business innovation and operations in a sustainable way. Most importantly, the associated side effects of blockchain project failure rates that create a negative impact on economic and social sustainability performances such as corporate governance, risk management, finance management, human resources, and culture management, and competitiveness can be operationalized, measured, and managed with the "TAM + CMM Adoption Model".

While we deem our results credible, there are certain limitations that we would like to elaborate upon. Our results represent a snapshot of the development, which were published until December 2021. Ongoing activities that are still not published or activities that have started during or after our research commenced are not reflected. Furthermore, there might be commercial studies conducted by consultancy firms, which are not available for the public at the time of writing. Some of the limitations that we plan to address are related to the need for continuing our case studies and further industry activities regarding the validation of the "TAM + CMM Adoption Model". We are currently structuring such industry-wide case studies to include the integration of each presented risk and success factor across the "TAM" and "CMM" levels.

Another dimension of our future research activities will include the conduction of post-pandemic research to identify the current state of blockchain adoption across various industries or focus on one specific industry, which is highly relevant for business innovation. The aftershocks of the worldwide pandemic response are affecting fundamental societal norms. This serves as a catalyst for the fast-paced adoption of technology and will make preand post-pandemic comparisons particularly interesting. We have identified the culture and creative industry as a possible sector for these future research activities. Based on these results, we will then consider how to extend the explanatory power of the "TAM + CMM Adoption Model" across higher maturity levels and other TAM levels. 


\section{Conclusions}

In general, the topic of blockchain technology in the context of business innovation is relevant, which is shown by high awareness levels. However, a strong decline in "TAM" parameters (level of knowledge, perceived usefulness, perceived ease of use, intention to use, active system use) across the "CMM" maturity levels, regardless of industry sector or company size, indicates that there is a substantial gap in managing the blockchain adoption process in a sustainable way. Based on the descriptive and empirical analysis, we conclude that the "TAM + CMM Adoption Model" seems to be a resilient and valuable model for identifying acceptance and maturity levels. Moreover, the applicability of the framework of the adoption model can be qualified by the very high survey closure rate of $98.5 \%$ of respondents.

The customized "TAM + CMM Adoption Model" holds the potential to support the sustainable usage of blockchain technology for business innovations, not limited to a specific industry or use case. The integrated "TAM + CMM Adoption Model" is capable of operationalizing blockchain-driven business innovation across all industries and use cases, including business models, products, services, supply-chain management, and more. This is demonstrated by the extent of industries and company sizes that participated in the empirical study.

We further conclude that the integration of "TAM" and "CMM" yields the following benefits and value propositions for business innovation: (1) simultaneously analyzing and managing the adoption dimensions of novelty and complexity; (2) simultaneously analyzing and managing acceptance and maturity as mission-critical aspects of those dimensions; (3) analyzing and managing risks related to each expression throughout the innovation process.

By merging the "TAM" and "CMM" parameters, the levels of acceptance can be observed at each maturity level. Thus, this model serves as a dynamic approach for managing risk and success factors. If risk parameters can be managed and influenced at the right time of the innovation process, maturity levels of blockchain adoption can be increased in a sustainable way. Economic and social sustainability performances such as corporate governance, risk management, finance management, human resources, culture management, and competitiveness can be operationalized, measured, and managed with the "TAM + CMM Adoption Model".

In the context of competitiveness, there is a verifiable risk for companies to lose momentum at the industry level due to the lack of an appropriate adoption model and sufficient approach to business innovation. Such risk could negatively impact competitive advantage and sustainable growth within their industry sector. This indicates that further education and immersion into blockchain-driven business innovation economy are required, and this novel adoption model could offer a valuable solution for this need.

It shall be highlighted that the "TAM + CMM Adoption Model" not only holds value for blockchain-driven business innovation but also for other emerging technologies, such as nanotechnology, AI, robotics, virtual reality, and the metaverse. Our contemporary era is characterized by the simultaneous rise of several foundational, emerging technologies, which possess relatively high degrees of novelty and complexity. The "TAM + CMM Adoption Model" can be considered technology agnostic, meaning it can also be applied for these other technologies that are capable of creating business innovation. In this context, the risk and success criteria would need to be amended according to the applied technology.

In closing, we conclude that there is a great opportunity to design and develop a "TAM + CMM Adoption Model" software-as-a-service (SaaS) application. Large user groups would be empowered to use this application to operationalize, measure and manage innovation processes across any industry or use case. Looking at the social sustainability aspect of community development, the captured data of the SaaS application could be made available to business innovation communities in order to learn from each other and to grow with each other. 
Author Contributions: Conceptualization, N.D, methodology, N.D. and V.S., data gathering, N.D., data analysis, N.D. and V.S., validation, D.A.A. All authors have read and agreed to the published version of the manuscript.

Funding: This research received no external funding.

Conflicts of Interest: The authors declare no conflict of interest.

\section{Appendix A}

\section{Questionnaire:}

Q1. What is your name?

Q2. Which company are you working for?

Q3. What is your position/role in your company?

Q4. Which size is your company?

Q5. Which industry does your company belong to?

Q6. Did you hear about blockchain technology?

Q7. When did you hear first about blockchain technology?

Q8. In which context did you hear about blockchain technology?

Q9. Are you aware of a comprehensive, structured methodology that could help you apply blockchain technology for your business?

Q10. Are you aware which value blockchain technology could create for your business?

Q11. Do you know what blockchain is and how it functions?

Q12. How good is your knowledge about blockchain technology?

Q13. Which value can you create for your business with blockchain technology?

Q14. Is your company actively using a comprehensive, structured methodology that helps you apply (create value) blockchain technology for your business?

Q15. How satisfied are you with the methodology, which your company is using to apply blockchain technology?

Q16. Do you have any active blockchain initiatives in your company?

Q17. Do you apply a comprehensive, structured methodology when initiating a blockchain project in your company?

Q18. How satisfied are you with the methodology, which your company is using to initiate blockchain technology?

Q19. Are you planning any blockchain initiatives in your company in the next 12 months?

Q20. Do you have any active blockchain implementation projects in your company?

Q21. Do you apply a comprehensive, structured methodology when implementing a blockchain project in your company?

Q22. How satisfied are you with the methodology, which your company is using to implement blockchain technology?

Q23. Are you planning any blockchain implementation projects in your company in the next 12 months?

Q24. Do you offer standardized products and services based on blockchain technology in your company?

Q25. Do you apply a comprehensive, structured methodology to standardize blockchain products and services in your company?

Q26. How satisfied are you with the methodology, which your company is using to standardize blockchain products and services in your company?

Q27. Are you planning to standardize blockchain products and services in your company in the next 12 months?

Q28. Do you scale standardized blockchain products and services in your company?

Q29. Do you apply a comprehensive, structured methodology to scale standardized blockchain products and services in your company?

Q30. How satisfied are you with the methodology, which your company is using to scale standardized blockchain products and services in your company? 
Q31. Are you planning to scale standardize blockchain products and services in your company in the next 12 months?

Q32. Do you optimize standardized and scaled blockchain products and services in your company?

Q33. Do you apply a comprehensive, structured methodology to optimize scaled and standardized blockchain products and services in your company?

Q34. How satisfied are you with the methodology, which your company is using to optimize standardized and scaled blockchain products and services in your company?

Q35. Are you planning to optimize standardized and scaled blockchain products and services in your company in the next 12 months?

\section{References}

1. Dess, G.; Pickens, J.C. Changing roles: Leadership in the 21st century. Organizational Dynamics. Open J. Bus. Manag. 2000, 28, 18-34.

2. Drljevic, N.; Arias Aranda, D.; Stantchev, V. Perspectives on Risks and Standards that Effect the Requirements Engineering of Blockchain Technology. Comput. Stand. Interfaces 2020, 69, 11-13. [CrossRef]

3. Bitcoinist. 92\% of Blockchain Projects Have Already Failed, Average Lifespan of 1.22 Years. Available online: https://bitcoinist. com/92-blockchain-projects-already-failed-average-lifespan-1-22-years/ (accessed on 10 December 2021).

4. Coinopsy. List of Dead Coins. 2021. Available online: https://www.coinopsy.com/dead-coins/ (accessed on 18 December 2021).

5. Forbes. Why Enterprise Blockchain Fail. Available online: https://www.forbes.com/sites/dantedisparte/2019/05/20/whyenterprise-blockchain-projects-fail/?sh=3fc907614b96 (accessed on 18 December 2021).

6. Compare Camp. Cryptocurrency Statistics. Available online: https://comparecamp.com/cryptocurrency-statistics/ (accessed on 10 December 2021).

7. Statista. Number of Blockchain Wallett Users Worldwide. Available online: https://www.statista.com/statistics/647374 /worldwide-blockchain-wallet-users / (accessed on 15 December 2021).

8. Gartner. 90\% of Current Enterprise Blockchain Platform Implementations Will Require Replacement by 2021. Available online: https:/ / www.gartner.com/en/newsroom/press-releases/2019-07-03-gartner-predicts-90--of-current-enterprise-blockchain (accessed on 10 December 2021).

9. Iansiti, M.; Lakhani, K. The truth about blockchain. Harv. Bus. Rev. 2017, 95, 118-127.

10. Alomary, A.; Woollard, J. How is technology accepted by users? A review of technology acceptance models and theories. In Proceedings of the IRES 17th International Conference, London, UK, 21 November 2015; pp. 1-3.

11. Wendler, R. The maturity of maturity model research: A systemic mapping study. Inf. Softw. Technol. 2012, 54, 1337-1339. [CrossRef]

12. OECD Blockchain Primer. Available online: http://www.oecd.org/finance/OECD-Blockchain-Primer.pdf (accessed on 24 November 2021).

13. IEEE SA Standards Associantion. Available online: https://standards.ieee.org/standard/29148-2018.html (accessed on 2 December 2021).

14. Science Direct. Blockchain Applicatons and Adoption Models. Available online: https://www.sciencedirect.com/search?qs= blockchain\%20applications\%20and\%20adoption\%20model\&lastSelectedFacet=years (accessed on 27 December 2021).

15. Science Direct. Sustainable Blockchain. Available online: https://www.sciencedirect.com/search?qs=sustainable\%20blockchain\& show $=100$ (accessed on 27 December 2021).

16. Yan, W.; Tan, T.-E.; Movva, P.V.H.; Fang, A.H.S.; Yeo, K.-K.; Ho, P.H.; Foo, F.S.S.; Xiao, Z.; Sun, K.; Wong, P.T.Y.; et al. Blockchain Applications in Health Care for COVID-19 and Beyond: A Systematic Review. Lancet Digit. Health 2021, 3, E819-E829.

17. Balci, G.; Surucu, E. Blockchain Adoption in the Maritime Supply Chain: Examining Barriers and Salient Stakeholders in Containerized International Trade. Transp. Res. Part E Logist. Transp. Rev. 2021, 156, 102539. [CrossRef]

18. Li, X.; Lai, P.; Yang, C. Determinants of Blockchain in the Aviation Industry. J. Air Transp. Manag. 2021, 97, 8. [CrossRef]

19. Safeena, R.; Date, H.; Hundewale, N.; Kammani, A. Combination of TAM and TPB in internet banking adoption. Int. J. Comput. Theory Eng. 2013, 5, 146-150. [CrossRef]

20. Orji, I.; Kusi-Sarpong, S.; Huang, S.; Vazquez-Brust, D. Evaluating the Factors That Influence the Blockchain Adoption in the Freight Logistics Industry. Transp. Res. Part E Logist. Transp. Rev. 2020, 141, 102025. [CrossRef]

21. Uddin, M.; Stranieri, A.; Gondal, I.; Balasubramanian, V. A Survey in the Adoption of Blockchain in IoT Challenges and Soutions. Blockchain Res. Appl. 2021, 2, 100006. [CrossRef]

22. Cuofano, G. What Is a Technology Adoption Curve? Four Week MBA. Available online: https:/ / fourweekmba.com/technologyadoption-curve/ (accessed on 25 November 2021).

23. Ridwandono, D.; Pribabi, A. IT and Organizational Agility: A Critical Literature Review. In Proceedings of the Fifth Information Systems International Conference, Surabaya, Indonesia, 23-24 July 2019; p. 155.

24. Goksen, Y.; Cevikb, E.; Avundukc, H. A case analysis on the focus on the maturity models and information technologies. Procedia Econ. Financ. 2015, 19, 208-216. [CrossRef] 
25. Wang, H.; Chen, K.; Xu, D. A maturity model for blockchain adoption. Financ. Innov. 2016, 2, 12. [CrossRef]

26. Edison, H.; Ali, N.B.; Torkar, R. Towards innovation measurement in the software industry. J. Syst. Softw. 2014, 86, 1390-1407. [CrossRef]

27. Crossan, M.; Apaydin, M. A multi-dimensional framework of organizational innovation: A systematic review of the literature. $J$. Manag. Stud. 2010, 47, 1154-1191. [CrossRef]

28. KPMG. Blockchain Maturity Model. Helping You to Get from Proof-of-Concept to Production; KPMG: Amsterdam, The Netherlands, 2018; Available online: https://assets.kpmg/content/dam/kpmg/nl/pdf/2017/advisory/blockchain-maturity-model.pdf (accessed on 24 November 2021).

29. Momani, A.; Jamous, M. The evolution of technology acceptance theories. Int. J. Comput. Corp. Res. 2017, 1, 50-58.

30. Rogers, E. The Diffusion of Innovation; Free Press, A Division of Simon \& Schuster: New York, NY, USA, 2003 ; Volume 5.

31. Davis, F.; Bagozzi, R.; Warshaw, P. Extrinsic and intrinsic motivation to use computers in the workplace. J. Appl. Soc. Psychol. 1992, 22, 1111-1132. [CrossRef]

32. Lou, A.; Li, E.Y. Integrating innovation diffusion theory and the technology acceptance model: The adoption of blockchain technology from business managers' perspective. In Proceedings of the ICEB 2017 Proceedings, Dubai, United Arab Emirates, 4-8 December 2017; p. 44.

33. Lee, Y.H.; Hsieh, Y.C.; Hsu, C.N. Adding innovation diffusion theory to the technology acceptance model: Supporting employees intentions to use e-learning systems. J. Educ. Technol. Soc. 2011, 14, 124-137.

34. Mathieson, K. Predicting user intentions: Comparing the technology acceptance model with the theory of planned behavior. Inf. Syst. Res. 1991, 2, 173-191. [CrossRef]

35. Al-Suqri, M.N.; Rahma Mohammed Al-Kharusi, R.M. Ajzen and Fishbein's Theory of Reasoned Action (TRA) (1980). In Information Seeking Behavior and Technology Adoption: Theories and Trends; Information Science Reference, Global: Hershey, PA, USA, 2005; pp. 188-204.

36. Agarwal, R. Individual Acceptance of Information Technologies. In Framing the Domains of IT Management Research; Zmud, R.W., Ed.; Pinnaflex Educational Resources, Inc.: Hawthorne, MI, USA, 2000; pp. 85-104.

37. Chen, L.D.; Gillenson, M.L.; Sherrell, D.L. Enticing online consumers: An extended technology acceptance perspective. Inf. Manag. 2002, 39, 705-719. [CrossRef]

38. Silva, P. Davis' Technology Acceptance Model (TAM) (1989). In Information Seeking Behavior and Technology Adoption: Theories and Trends; Al-Suqri, M.N., Al-Aufi, A.S., Eds.; Information Science Reference: Hershey, PA, USA, 2015; pp. 205-219.

39. Proença, D.; Borbinha, J. Maturity models for information systems-A state of the art. Procedia Comput. Sci. 2016, 100, 1042-1049. [CrossRef]

40. Park, S.H.; Eo, J.; Lee, J.J. Assessing and managing an organization's green IT maturity. MIS Q. 2012, 11, 127-140.

41. Rathore, S.; Panwar, A. Factors influencing behavioural intention to use smart phones. Glob. Vistas 2015, 2, 19-28.

42. Kim, K.; Kang, T. Does Technology Against Corruption Always Lead to Benefit? The Potential Risks and Challenges of the Blockchain Technology. In Proceedings of the OECD Global Anti-Corruption and Integrity Forum, Paris, France, 30-31 March 2017; pp. 12-15.

43. Caron, F. Blockchain: Identifying risks on the road to distributed ledgers. ISACS J. 2017, 5, 1-6.

44. Panchev, K. Why Today's Blockchain Can't Be Mainstream. Trending Topics Bulgaria. Available online: https://www. trendingtopics.at/bulgaria/why-todays-blockchain-cannot-be-mainstream/ (accessed on 10 October 2021).

45. Toufaly, E.; Zalan, T.; Dhao, S. A Framework of Blockchain technology Adoption: An Investigation of Challenges and Excpected Value. Inf. Manag. 2021, 58, 10. [CrossRef]

46. MIT Sloan Management Review. Customer Focused KPIs Fuel the Future of Business. Available online: https://sloanreview.mit. edu/customer-focused-kpis-fuel-the-future-of-business / (accessed on 25 November 2021).

47. Kasunic, M.; Anderson, W. Measuring Systems Interoperability: Challenges and Opportunities; Carnegie Mellon University: Pittsburgh, PA, USA, 2014; p. 2. 